\title{
Effects of Training and Detraining on Physical Fitness, Physical Activity Patterns, Cardiovascular Variables, and HRQoL after 3 Health-Promotion Interventions in Institutionalized Elders
}

\author{
Alexandrina Lobo, Joana Carvalho, and Paula Santos \\ Research Center in Physical Activity, Health and Leisure, Faculty of Sport, University of Porto, Rua Dr. Plácido Costa 91, \\ 4200-450 Porto, Portugal \\ Correspondence should be addressed to Alexandrina Lobo, damiaolobo@gmail.com
}

Received 30 July 2010; Revised 20 December 2010; Accepted 21 December 2010

Academic Editor: Samuel Y. S. Wong

Copyright ( $\odot 2010$ Alexandrina Lobo et al. This is an open access article distributed under the Creative Commons Attribution License, which permits unrestricted use, distribution, and reproduction in any medium, provided the original work is properly cited.

\begin{abstract}
The purpose of this study is to assess the effects of different strategies of health on the levels of physical activity (PA), physical fitness $(\mathrm{PF})$, cardiovascular disease (CVD) risk factors and quality of life (QoL) of the institutionalized elderly. Concurrently studies were made of the effect of detraining on these same variables. In this investigation we carried out a prospective longitudinal study with an experimental design, with 1 year plus 3 months of a detraining period. Methodology. (a) A questionnaire with sociodemographic characteristics and a QoL scale (MOS SF-36); (b) Functional Fitness Test to assess PF; (c) An MTI Actigraph to evaluate the PA; (d) Biochemical analysis of blood, blood pressure and bio-impedance. The Main Results Indicated That: (i) ST significantly improved strength and body flexibility and AT the aerobic endurance, agility/dynamic balance and lower strength and flexibility; (ii) Implications of detraining were more evident on the PA groups in the lower body flexibility, which is associated with agility/dynamic balance and lower strength in the AT group; (iii) Cardiovascular variables improved significantly especially blood pressure, cholesterol and glucose in the ST and HDL in the AT group; not having undergone significant changes with the detraining. The results of this thesis contribute positively to highlight the importance of PA in the promotion of health, prevention and reduction of CVD risk factors and the improvement of the PF and QoL.
\end{abstract}

\section{Introduction}

In developed countries, people aging 65 and older constitute approximately $20 \%$ of the population and are the fastestgrowing segment of the population [1]. With this growing aging population, the number of institutionalization will likely increase in coming years. Being relocated to an assisted living facility can result in functional and health disturbances and worsen quality of life in elders. This may be attributed to or worsened by lack of regular physical activity. Appropriate exercise programs may be an important component of quality of life in this group of transitional frail elders $[2,3]$.

Previous studies suggest that participation in a regular exercise program can be an effective intervention to reduce/prevent a number of functional declines associated with aging $[4,5]$. In this regard, current guidelines point out the importance of aerobic exercise in the maintenance and improvement of various aspects of cardiovascular function and health and strength training in the attenuation of muscle strength declines associated with normal aging $[6,7]$.

Moreover, considering that cardiovascular diseases (CVD) are the major cause of death in the elders, sustaining older adults' ability to live independently as well as reducing blood pressure (BP), plasma lipoprotein lipid profiles, and body weight via healthy lifestyles interventions are very important goals of public health, geriatrics, and gerontology. Although there is sufficient data showing that regular physical activity (PA) is associated with improvements in cardiovascular health among older adults [8-10], additional information is necessary concerning the effectiveness of different types of heath promotion and exercise interventions. 
Furthermore, regarding that a number of studies have indicated that both morphologic and functional adaptations can decrease even after short detraining periods [11], it seems of interest to know whether the heath promotion interventions benefits are maintained when detraining occurs, due to unexpected causes such as, illness, vacations, or others. In fact, despite evidence of physiological decline during detraining, there is not enough data suggesting how long the beneficial effects of training are maintained, how this affects the health related quality of life (HRQoL) and how functional fitness changes following the cessation of exercise intervention in institutionalized elders [12].

Most studies of detraining in elders have examined the effects after exercise intervention and focused on different performance measures of physical function, resembling the effects on functional fitness response [13-15]. While some studies have shown similar decreases in strength, aerobic endurance, and agility/dynamic balance $[11,16]$, others have showed that increases in muscle strength after training can be maintained after 24 weeks [11], 27 weeks [17], and 31 weeks [18] of detraining in older adults. According to the literature the initial levels of physical activity and functional capacity, gender differences, duration of the detraining period, age, and the evaluation method used could be possible explanations for the discrepancy between studies. Few studies have reported detraining evidence after different types of interventions $[12,19]$ and those have not addressed HRQoL and cardiovascular health variables.

Therefore, the purposes of this study were to investigate the effects of 1 year of 3 different health intervention programs (aerobic training, strength training, and health education) on physical fitness, PA patterns, cardiovascular health variables, and HRQoL. In addition, the effect of 3 months of detraining was also analysed.

\section{Methods}

2.1. Subjects. Subjects were recruited from 8 long-term care institutions (nursing homes) randomly selected in the Oporto area (North of Portugal). Two hundred nineteen elderly subjects aging 66 to 92 volunteered to participate in this study. At the screening, participants completed a health history questionnaire to record past and present conditions and medication, as well as a sociodemographics and years of institutionalization questionnaire. Participants were considered eligible for inclusion if they had scored 24 or more on the mini-mental state examination (without mental dysfunction) [20] with a Barthel index 40 or more (without several dependence) [21] and also if they were not engaged in regular exercise of moderate to vigorous intensities for 20 minutes or more at least twice a week in the past 3 years [22]. Participants were excluded if they had any medical or physical limitations for testing or training and were without medical assistance or control drugs. There were 11 elders with osteoporosis, 24 with diabetes, 7 with respiratory illness, 19 with hypertension, 44 with hypercholesterolemia, and 5 with arthritis. In general, each subject received 6 different drugs (range 4-11) daily, including supplements such as calcium, vitamin B, diuretics, laxatives, heart drugs, antihypertensive, antidepressants, and painkillers.

Before conducting the study, all participants received a complete explanation of the purpose, risks, and procedures of the investigation and gave their written consent. The investigation was in full compliance with the Helsinki declaration of 1975, as revised in 2004. All methods and procedures were approved by the Institutional Review Board. The elderly subjects provided written informed consent before the study.

2.2. Design. Due to recruitment implications according to infrastructures available on each institution, this study was not a true randomized controlled trial. Therefore, the 185 eligible subjects, from institutions registered in this study, were allocated into four groups: aerobic exercise program (AT; $N=49$ ), strength training program (ST; $N=37$ ), health education program (HE; $N=52$ ), and control group (CG; $N=47$ ). However, after randomization, 19 participants of the ST group volunteered for the control group, potentially reducing the sample size of ST program $(N=18)$.

Data were collected by trained investigators, using always the same protocol, for all variables at baseline (M1-January 2006), after the 1-year control period (M2-February of 2007), and, finally, after 3 months of detraining (M3-May of 2007).

\section{Health-Promotion Interventions}

3.1. Exercise Interventions. The 1-year exercise interventions were held twice a week, each session lasted 30-40 minutes and were supervised by a qualified and trained instructor.

3.2. Aerobic Training. Briefly, AT sessions consisted of 10 minutes warm-up, followed by 20 minutes of aerobic workout using continuous walking and dancing using rhythmic and large muscle groups movements and ended with a 810 minutes of cool-down. In the first month, duration was increased from 20 to 30 minutes with an intensity of 2-3 of the adapted Borg Rating of Perceived Exertion scale (RPE) [23]. Subsequently, the intensity was gradually increased up to 4-5 in the adapted Borg RPE. Moreover, in order to evaluate the intensity of AT and detect workloads that rise above moderate intensity, the talk test was also used.

3.3. Strength Training. ST consisted of 2 sets of 6 exercises (each with 8 to 12 repetitions) specifically directed to the development of endurance, muscle mass, and strength of the quadriceps (using leg press and leg extension), hamstrings (using seated leg curl), trunk and arms (using women's double chest, lateral raise, and overhead press), and abdominal wall (using an abdominal machine). Movements were repeated approximately every 6 seconds, with 2 seconds for each lifting movement (concentric muscle action) and 4 seconds for each lowering movement (eccentric muscle action). During the first two weeks the training intensity was $45-50 \%$ of $1 \mathrm{RM}$ in order to promote adaptation to exercise routine. From third week, the load was raised to $60-65 \%$ of 
$1 \mathrm{RM}$ and maintained until the final of the program. The 1 RM tests were performed each 15 days until the first month and then each 4 weeks until the end of the program.

3.4. Health Education. For the elderly receiving HE, health professionals provided encouragement and reinforcement of the importance about PA without providing any exercise protocols to follow. These interventions covered broad domains of health care management, well-being and social awareness related to elderly people's health, and health care. Health care management was primarily related to nutritional advice, management of bone, and joint-related illness (e.g., joint pain, arthritis, and back pain) as well as other illnesses (e.g., diabetes, high blood pressure, etc.). An expositive and participative strategy was adopted for 1 hour per week sessions.

3.5. Detraining. Normal activities of daily living were maintained during detraining, but none of the subjects participated in any supervised exercise program. In order to control the absence of any exercise or other healthpromotion interventions, they were contacted face to face by the health professionals of the institutions.

3.6. Control Group. Subjects allocated into CG $(N=66)$ were instructed not to change their daily living PA routines or dietary patterns during the course of the study and not to carry out any supervised PA and were informed that they would receive intervention after the end of the study. After the observation period, subjects randomized to the CG were invited to participate in specific exercise programs designed to seniors undergoing in the institution.

\section{Measurements}

\subsection{Study Variables}

4.1.1. Anthropometrics. Body mass index (BMI) measure was used as an estimate of body composition. Body height was measured to an accuracy of $1 \mathrm{~cm}$, with the subject in an upright position with a standard stadiometer. Body weight was measured to the nearest $0.1 \mathrm{~kg}$, with subjects lightly dressed and in stocking feet. BMI was calculated using the standard formula: mass $(\mathrm{kg}) /$ height $\left(\mathrm{m}^{2}\right)$. The percentage of body fat was evaluated with the bioelectrical impedance Bodystat it's the same that Stella [23]. This is an alternative easy and noninvasive method to specifically assess the percentage of fat through a low-intensity electric current that runs through the subject's body [24]. The average values of body fat were between 17 and $21 \%$ for men and 22 and $31 \%$ for women [25].

4.1.2. Subjective Health-Related Quality of Life. Older adults' perceptions of their general health were assessed using the Medical Outcomes Study 36-Item Short-Form Health Study-MOS SF-36 adapted and validated for Portuguese population [26]. The MOS SF-36 is a generic measure of health condition including 36 items covering 8 dimensions: physical functioning (PF-10 items), body pain perception (BP-2 items), general health ( $\mathrm{GH}-5$ items), vitality
(VT-4 items), social functioning (SF-2 items), emotional role limitations ( $\mathrm{EH}-3$ items), limitations due to physical health $(\mathrm{PH})$, and mental health $(\mathrm{MH}-5$ items) scores are summarized into two main domains (physical and mental component) and ranged from 0 (worst possible quality of life) to 100 (best possible quality of life). As suggested by Ware and Sherbourne [27], the instrument was applied by an interview and scores were calculated using the methods set out by the authors.

4.1.3. Physical Fitness. Physical fitness was evaluated using the functional fitness test (FFT) [28], that consists of 6 validated items (and one alternative) designed to assess the physical mobility of older adults.

Lower body strength was measured using the 30 -second chair stand test. Participants were asked to sit on a $43-\mathrm{cm}-$ high chair with arms crossed at the wrists and held against the chest. Participants completed as many "stand ups" as possible within $30 \mathrm{~s}$. The score was the total number of stands executed through the full range of motion within $30 \mathrm{~s}$. The reliability of this strength test is high $(r=0.92)$ [28].

Upper body strength was assessed using the arm curl test. Participants performed as many biceps curls as possible in $30 \mathrm{~s}$, using a dumbbell. The score was the total number of handweight curls performed through the full range of motion in $30 \mathrm{~s}$. The arm curl test has good relative reliability across trials $(r=0.80)$ [28].

Lower body flexibility was assessed using the chair sitand-reach test. The score was the best distance achieved between the extended fingers and the tip of the toe, measured to the nearest $0.5 \mathrm{~cm}$. The reliability of the chair sit-and-reach test is high $(r=0.96)$ [28].

Upper body flexibility was assessed using the back scratch test. The score was the shortest distance achieved between the extended middle fingers, measured to the nearest $0.5 \mathrm{~cm}$. The reliability of this test is high $(r=0.92)$ [28].

Agility/dynamic balance was assessed using the 8 -foot up-and-go test. The score was the shortest time to rise from

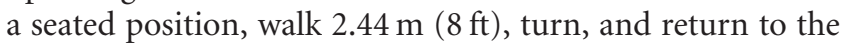
seated position, measured to the nearest $1 / 10$ th $\mathrm{s}$. The 8 -foot up-and-go test has showed a high test-retest reliability of $0.90[28]$.

Aerobic endurance was measured using the 6-minute walk test. Participants were asked to walk as fast as possible for 6 min with verbal encouragement given at 30-second intervals. The score was the total distance walked in $6 \mathrm{~min}$ along a 45.72-meter rectangular course, which was marked every $4.57 \mathrm{~m}$. The reliability of this test is high $(r=0.91)$ [28].

All test stations were organized in a circuit and the same conditions were maintained for each test at all testing periods, after a demonstration by the tester, a practice trial of 2 repetitions, followed by 1 test trial. Full detailed information on test administration and protocols can be found in the work of Rikli and Jones [28].

4.1.4. Blood Pressure. Resting blood pressure (BP) was measured with the Dinamap vital signs monitor (Critikon; GE Medical Systems, Milwaukee, WI), using the right 
TABLE 1: Baseline, postintervention, and postdetraining values for cardiovascular variables and delta changes.

\begin{tabular}{|c|c|c|c|c|c|c|c|c|c|}
\hline \multirow{2}{*}{ Age (years) } & & \multirow{2}{*}{$\begin{array}{c}\mathrm{CG}(n=51) \\
77.6 \pm 6.9\end{array}$} & \multicolumn{3}{|c|}{$\mathrm{AT}(n=35)$} & \multirow{2}{*}{$\begin{array}{c}\mathrm{ST}(n=10) \\
75.1 \pm 8.3\end{array}$} & \multicolumn{3}{|c|}{$\mathrm{HE}(n=41)$} \\
\hline & & & $\%$ Change & $81.0 \pm 7.1$ & $\%$ Change & & $\%$ Change & $81.6 \pm 6.7$ & $\%$ Change \\
\hline \multirow{3}{*}{$\begin{array}{l}\text { PA } \\
\text { (counts/h) }\end{array}$} & B- $\Delta_{1}$ & $11591 \pm 7739$ & -5.8 & $10953 \pm 6219$ & 62.4 & $11938 \pm 7920$ & 43.1 & $12091 \pm 8519$ & 15.3 \\
\hline & $\mathrm{AI}-\Delta_{2}$ & $10915 \pm 7112$ & & $17761 \pm 8218^{* a}$ & -13.1 & $17053 \pm 5519^{* a}$ & -1.7 & $13933 \pm 6902$ & -1.8 \\
\hline & $\mathrm{AD}-\Delta_{3}$ & & & $15461 \pm 8008$ & 41.5 & $16756 \pm 8765$ & 40 & $13676 \pm 7861$ & 13.1 \\
\hline \multirow{3}{*}{$\begin{array}{l}\text { SBP } \\
(\mathrm{mmHg})\end{array}$} & B- $\Delta_{1}$ & $138.8 \pm 11$ & -0.7 & $133.4 \pm 22$ & -1.3 & $130.3 \pm 15$ & -3.7 & $128.5 \pm 13$ & -1.5 \\
\hline & AI $-\Delta_{2}$ & $139.3 \pm 12$ & & $131.6 \pm 17$ & -2.5 & $125.1 \pm 6$ & 2.8 & $126.5 \pm 13$ & -2.3 \\
\hline & $\mathrm{AD}-\Delta_{3}$ & & & $128.3 \pm 17$ & -3.8 & $128.6 \pm 16$ & -1.4 & $123.5 \pm 14$ & -3.9 \\
\hline \multirow{3}{*}{$\begin{array}{l}\text { DBP } \\
(\mathrm{mmHg})\end{array}$} & B- $\Delta_{1}$ & $80.7 \pm 9.4$ & -0.8 & $82 \pm 10$ & -2.4 & $80.5 \pm 11$ & -2.1 & $76.9 \pm 10.2$ & -1.3 \\
\hline & AI $-\Delta_{2}$ & $80.0 \pm 9.6$ & & $80 \pm 4 \pm 9$ & 0.8 & $78.8 \pm 15$ & -0.4 & $75.9 \pm 11$ & 3.7 \\
\hline & $\mathrm{AD}-\Delta_{3}$ & & & $80.7 \pm 11$ & -1.5 & $78.4 \pm 8$ & -2.6 & $78.7 \pm 10$ & 2.4 \\
\hline \multirow{3}{*}{ Cholesterol } & B- $\Delta_{1}$ & $181 \pm 32$ & 3.4 & $191 \pm 28^{\mathrm{a}}$ & -14.1 & $182 \pm 20^{\mathrm{a}}$ & -24 & $178 \pm 18$ & -2.2 \\
\hline & $\mathrm{AI}-\Delta_{2}$ & $187.3 \pm 36$ & & $164 \pm 37$ & 4.2 & $138 \pm 23^{b}$ & 5.1 & $174 \pm 18$ & -1.7 \\
\hline & $\mathrm{AD}-\Delta_{3}$ & & & $171 \pm 24$ & -10.2 & $145 \pm 31$ & -20.2 & $171 \pm 13$ & -3.9 \\
\hline \multirow{3}{*}{$\begin{array}{l}\mathrm{HDL} \\
(\mathrm{mg} / \mathrm{dL})\end{array}$} & B- $\Delta_{1}$ & $51.2 \pm 9.6$ & -2.5 & $48.9 \pm 7.1$ & 4.2 & $53.6 \pm 12$ & -0.7 & $55.6 \pm 12$ & -1.9 \\
\hline & AI- $\Delta_{2}$ & $49.9 \pm 9.8$ & & $50.9 \pm 11$ & -5.5 & $53.2 \pm 6$ & -5.8 & $54.5 \pm 10$ & 0.0 \\
\hline & $\mathrm{AD}-\Delta_{3}$ & & & $48.1 \pm 8$ & -1.6 & $50.1 \pm 5$ & -6.5 & $54.5 \pm 10$ & -1.9 \\
\hline \multirow{3}{*}{$\begin{array}{l}\text { Glucose } \\
(\mathrm{mg} / \mathrm{dL})\end{array}$} & B- $\Delta_{1}$ & $108 \pm 30$ & 0.9 & $106 \pm 28$ & -2.7 & $115 \pm 39$ & -11.2 & $107 \pm 31$ & 0.1 \\
\hline & $\mathrm{AI}-\Delta_{2}$ & $109 \pm 24$ & & $103.1 \pm 22$ & 0.6 & $102 \pm 17$ & 0.8 & $107 \pm 29$ & -6.5 \\
\hline & $\mathrm{AD}-\Delta_{3}$ & & & $103.8 \pm 32$ & -2.1 & $103 \pm 12$ & -10.4 & $100 \pm 15$ & -6.6 \\
\hline \multirow{3}{*}{ HRQoL } & B- $\Delta_{1}$ & $62.0 \pm 13.4$ & -6.6 & $64.9 \pm 14.2$ & 8.2 & $60.1 \pm 15.1$ & 13.8 & $58.3 \pm 12.6$ & 5.2 \\
\hline & AI- $\Delta_{2}$ & $57.9 \pm 9.8$ & & $70.2 \pm 6.7$ & 0.8 & $68.4 \pm 11.1$ & -3.1 & $61.3 \pm 9.4$ & -3.5 \\
\hline & $\mathrm{AD}-\Delta_{3}$ & & & $69.5 \pm 7.5$ & 7.1 & $66.3 \pm 9.4$ & 10.1 & $59.2 \pm 10.4$ & 1.7 \\
\hline
\end{tabular}

CG: control group; AT: aerobic training; ST: strength training; HE: health education.

Univariate analysis of variance.

a Significant difference versus CG, $P<.05$.

${ }^{\mathrm{b}}$ Significant difference versus $\mathrm{HE}, P<.05$.

* Significant difference versus baseline, $P<.05$.

\# Significant difference versus after intervention, $P<.05$.

B: baseline; AI: after intervention; AD: after detraining.

Mixed-model ANOVA with repeated measures.

$\$$ Significant, $P<.05$.

arm. After being 15 minutes at supine rest in a quiet, temperature-controlled room, BP measurements were taken with the subjects seated in an upright position with the arm comfortably placed at heart level. Measurements of the diastolic (DBP) and systolic (SBP) BPs were taken within a 1-minute break, by the same investigator. The average of the 3 measurements for SBP and DBP was entered as data [29]. The measurements were performed between 8:00 AM and 11:00 AM, at least 30 minutes after meal. Furthermore, none of the subjects were smoker or under caffeine effect. Subjects were deemed to be hypertensive where their SBP was $\geq 140 \mathrm{mmHg}$ and their DBP was $\geq 90 \mathrm{mmHg}$ or they were on current antihypertensive drug treatment [30].

4.2. Biochemical Analyses. A venous blood sample for biochemical assays was withdrawn from the antecubital vein after an overnight fast for measurement of fasting blood glucose and lipid concentrations (high-density lipoprotein (HDL), low-density lipoprotein (LDL), triglycerides (TRIGs) and total cholesterol (TC)). Samples were stored at $80^{\circ} \mathrm{C}$ and analysed using the cholesterol esterase/oxidase enzymatic method, and triglyceride was analysed using the lipase/glycerol kinase/glycerol phosphate oxidase enzymatic method. HDL was analyzed using the homogeneous polyanion/cholesterol esterase/oxidase enzymatic method. Glucose was analyzed using the hexokinase method. Blood lipids and glucose were measured on an Olympus AU600 autoanalyser.

4.3. Daily Physical Activity. An accelerometer MTI Actigraph [31] was used before and after the intervention as an objective measure of daily PA over seven consecutive days. All participants were instructed about how to carry out the accelerometer that should be placed in a small nylon pouch and firmly adjusted at the person's waist using an elastic belt over the hip. A data sheet was given to each senior participant who was instructed to record the time when the monitor was attached in the morning and detached in the evening and every time he or she performed any restricted activities like showering and swimming. 
These data, summarized as counts per day, were considered to be complete if $70 \%$ of the day (1000 minutes) was recorded for at least 4 of the 5 week days, and 2 days of the weekend. For the present study, the time duration or sampling period was set at 1 minute and the output was expressed as counts per minute (counts. $\mathrm{min}^{-1}$ ). Then, for the statistical analysis we calculated the mean of the counts by hour. PA was also expressed as the average counts per hour (counts $\cdot \mathrm{h}^{-1}$ ) over the 7 days.

To the best of our knowledge, there are no appropriate count cut-points that represent meaningful intensity categories (sedentary, moderate, and vigorous) in older adults, therefore, in the current study, activity intensities were based according to the research findings of Freedson et al. [32].

4.4. Statistical Analyses. All data are reported as means $( \pm$ SD). Data were analysed with SPSS software (version 17.0). The level of significance was set at $P<.05$.

Shapiro-Wilk test was used to ascertain on all variables the normality of data distributions. The delta $(\Delta)$ was calculated via the standard formula: $\Delta=\{$ (posttest scorepretest score)/pretest score $\}$. Weight outcomes were assessed by three different moments: $\Delta_{1}$ change from baseline to 1 year, $\Delta_{2}$ weight change from 1 year to 15 months, and also $\Delta_{3}$ weight change from baseline to 15 months.

Differences within the group in pre- versus postintervention and detraining values were performed by one-way analysis of variance (ANOVA). Scheffe was used for post hoc comparisons. Repeated measures and multivariate analyses of variance were used to examine differences within and between groups over time. When $F$ values were significant, post hoc means comparisons were analysed with least significant different comparisons test.

\section{Results}

Of the 185 participants who submitted the initial assessment, $148(75 \%)$ participants completed pre- and postintervention and testing protocols. However, of these 148, only 137 completed the 15-month assessments (including the detraining period). During the study, 25 persons died, 11 moved out of the institutions, and 12 dropped out from the programs (Figure 1).

Overall compliance with the exercise prescription was $78 \%$ in the AT program and $60 \%$ in the ST program. Demographics and descriptive parameters of all groups are listed in Table 1. There were no differences among groups at baseline in relevant characteristics such as age, medical condition, BMI, fitness score, HRQoL, and PA.

Table 1 also shows that after the intervention, no significant changes were observed in the control group, although the trend indicated an increase in SBP, TC, and glucose levels. However, exercise interventions groups showed a significant increase in the PA levels and improve cardiovascular variables (glucose, TC levels, and blood pressure). Similar results occured in the EH group but without statistical significance. After the detraining, we did not observe significant change in cardiovascular variables and $\mathrm{PA}$, which remains significantly higher than baseline values.
Changes in functional fitness variables and body composition were presented in Table 2.

Our results showed that the biggest improvements of FFT were in the strength and flexibility tests in the ST group and in the aerobic endurance, lower body strength, and agility/dynamic balance in the AT group. The control group significantly decreases the agility/dynamic balance and the lower body strength and flexibility.

The implications of detraining in our subjects are most evident at lower-extremity body although, the upper body strength also decreased in the ST, nevertheless the values remained higher than before training. Only the agility/dynamic balance, particularly in the AT group, reverted to values lower than baseline, but not statistically significant.

\section{Discussion}

The overall results of this one-year health-promotion interventions study in institutionalized older subjects were the improvement in physical fitness, HRQoL, and cardiovascular variables, particularly in the exercise intervention groups. After the detraining, agility/dynamic balance was affected and also components from lower-extremity body, as strength and flexibility. The data also revealed a significant increase in the PA levels after intervention and declining with detraining, but remaining significantly higher than baseline values.

In accordance with the actual evidence, our results showed a major improvement in strength and flexibility in the ST group $[4,13,33]$ and a significant improvement in lower body strength, aerobic endurance, and agility/dynamic balance in the AT group [34].

Although, previous investigations have demonstrated an age-related functional decline, these improvements are not unexpected, because, as explained earlier in the literature the specificity of the training exists even in older subjects [5, $35,36]$. Further, our results also confirm the hypothesis that one year of moderate intensity exercise interventions (twice per week) is associated with several important cardiovascular risk factors and function, namely, the declining of BP and also benefits in the HRQoL.

Simons and Andel [34] showed that walking program is able to increase the aerobic endurance and flexibility and improve the performance of elders in the "sit-andreach test". In our study, the agility/dynamic balance improvements, particularly in the AT, might be related to increased lower body strength observed during the training period. According to Marzilli et al. [33] better balance was due to rises in lower body strength in older adults. Additionally, specific balance activities used in the present study may possibly have stimulated additional benefits. It was not possible to establish the independent effect of exercise programs on flexibility, because all of the programs also included flexibility exercises [37].

In this sense, Peterson et al. [7] state that the AT, as well as being used in the elderly, has excellent health benefits, increasing cardiofitness and strength and reduces the risk factors associated with CVD and metabolic diseases [38]. However to promote and maintain physical health 
TABLE 2: Baseline, postintervention, and postdetraining values of functional fitness test and delta changes.

\begin{tabular}{|c|c|c|c|c|c|c|c|c|c|}
\hline & & $\begin{array}{c}\text { CG } \\
(n=51)\end{array}$ & $\%$ Change & $\mathrm{AT}(n=35)$ & $\%$ Change & $\mathrm{ST}(n=10)$ & $\%$ Change & $\mathrm{HE}(n=41)$ & $\%$ Change \\
\hline \multirow{3}{*}{$\begin{array}{l}\text { Lower body } \\
\text { strength (no. in } \\
30 \mathrm{~s} \text { ) }\end{array}$} & B- $\Delta_{1}$ & $14.8 \pm 4.5$ & -12.5 & $14.6 \pm 4.7$ & 27.1 & $12.8 \pm 5.9$ & 25.8 & $13.8 \pm 5.4$ & 7.3 \\
\hline & $\mathrm{AI}-\Delta_{2}$ & $13.3 \pm 3.7$ & & $18.5 \pm 5.5^{* a}$ & -11.4 & $16.1 \pm 5.5^{*}$ & -7.4 & $14.9 \pm 4.4$ & 5.3 \\
\hline & $\mathrm{AD}-\Delta_{3}$ & & & $16.6 \pm 5.2^{\#}$ & 14.2 & $14.9 \pm 5.3^{\#}$ & 16.4 & $15.7 \pm 5.2$ & 13.7 \\
\hline \multirow{3}{*}{$\begin{array}{l}\text { Upper body } \\
\text { strength (no. in } \\
30 \mathrm{~s} \text { ) }\end{array}$} & B- $\Delta_{1}$ & $18.2 \pm 6.7$ & 1.1 & $20.7 \pm 9.9$ & 3.4 & $14.5 \pm 6.5$ & 49.6 & $17.3 \pm 9.8$ & 9.3 \\
\hline & $\mathrm{AI}-\Delta_{2}$ & $18.4 \pm 5.9$ & & $21.3 \pm 9.1$ & -1.3 & $21.7 \pm 7.2^{*}$ & -9.2 & $18.9 \pm 8.3$ & -7.4 \\
\hline & $\mathrm{AD}-\Delta_{3}$ & & & $21.1 \pm 8.9$ & 2.1 & $19.7 \pm 6.9^{\#}$ & 35.8 & $17.5 \pm 8.6$ & 1.2 \\
\hline \multirow{3}{*}{$\begin{array}{l}\text { Aerobic } \\
\text { endurance (no. } \\
\text { in } 2 \text { min) }\end{array}$} & B- $\Delta_{1}$ & $99.2 \pm 21.2$ & -7.4 & $102.3 \pm 21.9$ & 9.3 & $107.2 \pm 13.5$ & 4.4 & $96.7 \pm 19.7$ & 3.7 \\
\hline & $\mathrm{AI}-\Delta_{2}$ & $92 \pm 23.1^{*}$ & & $111.8 \pm 17^{* a}$ & -7.3 & $111.9 \pm 11.5^{\mathrm{a}}$ & 0.4 & $100.3 \pm 18.3$ & 6.1 \\
\hline & $\mathrm{AD}-\Delta_{3}$ & & & $104.1 \pm 18.7$ & 2.2 & $112.3 \pm 12.5$ & 4.8 & $106.4 \pm 18.9$ & 10.1 \\
\hline \multirow{3}{*}{$\begin{array}{l}\text { Lower body } \\
\text { flexibility (in. } \\
\text { from toe) }\end{array}$} & B- $\Delta_{1}$ & $-1.2 \pm 4.9$ & -125.2 & $-1.4 \pm 5.7$ & 21.4 & $-2.4 \pm 4.1$ & 45.8 & $-2.0 \pm 4.9$ & 5.1 \\
\hline & $\mathrm{AI}-\Delta_{2}$ & $-2.7 \pm 4.4^{*}$ & & $-1.1 \pm 4.8^{\mathrm{a}}$ & -27.3 & $-1.2 \pm 3.9^{*}$ & -25 & $-1.9 \pm 3.8$ & 6.7 \\
\hline & $\mathrm{AD}-\Delta_{3}$ & & & $-1.4 \pm 5.6^{\#}$ & 0.1 & $-1.5 \pm 4.0^{\#}$ & 37.5 & $-1.4 \pm 4.2$ & 0.3 \\
\hline \multirow{3}{*}{$\begin{array}{l}\text { Upper body } \\
\text { flexibility (from } \\
\text { fingers) }\end{array}$} & B- $\Delta_{1}$ & $-9.6 \pm 8.2$ & -1.4 & $-10.8 \pm 8.9$ & 15.7 & $-15.4 \pm 12.2$ & 22.7 & $-10 \pm 8.1$ & 1.1 \\
\hline & $\mathrm{AI}-\Delta_{2}$ & $-9.5 \pm 6.6$ & & $-9.1 \pm 6.5^{*}$ & -8.7 & $-11.9 \pm 8.7^{*}$ & -0.8 & $-9.9 \pm 7.1$ & -3.2 \\
\hline & $\mathrm{AD}-\Delta_{3}$ & & & $-9.9 \pm 6.9$ & 8.8 & $-12 \pm 9.9$ & 22.1 & $-10.2 \pm 7.8$ & -2.1 \\
\hline \multirow{3}{*}{$\begin{array}{l}\text { Agility/dynamic } \\
\text { balance (sec.) }\end{array}$} & B- $\Delta_{1}$ & $13.4 \pm 7.4$ & 11.4 & $12.7 \pm 7.7$ & -22.8 & $12.6 \pm 5.8$ & -9.5 & $13.2 \pm 6.9$ & 3.8 \\
\hline & $\mathrm{AI}-\Delta_{2}$ & $14.9 \pm 7.3$ & & $9.8 \pm 5.7^{* a b}$ & 41.8 & $11.4 \pm 4.7^{\mathrm{d}}$ & 9.6 & $13.7 \pm 5.2$ & 0.7 \\
\hline & $\mathrm{AD}-\Delta_{3}$ & & & $13.9 \pm 6.2^{\#}$ & 9.4 & $12.5 \pm 4.9$ & -0.8 & $13.8 \pm 5.8$ & 4.5 \\
\hline \multirow{3}{*}{$\mathrm{BMI}\left(\mathrm{Kg} / \mathrm{m}^{2}\right)$} & B- $\Delta_{1}$ & $26.9 \pm 3.1$ & 11.6 & $25.7 \pm 3.1$ & -8.9 & $24.6 \pm 0.2$ & 1.2 & $25.1 \pm 2.9$ & 13.9 \\
\hline & $\mathrm{AI}-\Delta_{2}$ & $29.8 \pm 3.4^{\mathrm{b}}$ & & $23.4 \pm 3.0^{\mathrm{ab}}$ & -2.1 & $24.9 \pm 3.1$ & 0.8 & $28.6 \pm 2.7$ & -2.8 \\
\hline & $\mathrm{AD}-\Delta_{3}$ & & & $22.9 \pm 4.1^{\mathrm{ab}}$ & -10.9 & $25.1 \pm 4.3$ & 2.1 & $27.8 \pm 3.2$ & 10.6 \\
\hline \multirow{3}{*}{$\begin{array}{l}\text { Body fat mass } \\
(\%)\end{array}$} & B- $\Delta_{1}$ & $22.1 \pm 5.8$ & 6.8 & $21.9 \pm 6.4$ & -9.6 & $22.5 \pm 8.8$ & 2.6 & $17.2 \pm 6.2$ & 16.8 \\
\hline & $\mathrm{AI}-\Delta_{2}$ & $23.6 \pm 4.1$ & & $19.8 \pm 5.6$ & 1.5 & $23.1 \pm 7.2$ & 1.7 & $20.1 \pm 7.6$ & 0.5 \\
\hline & $\mathrm{AD}-\Delta_{3}$ & & & $20.1 \pm 6.8$ & -8.1 & $23.5 \pm 8.6$ & 4.4 & $20.2 \pm 8.4$ & 17.4 \\
\hline
\end{tabular}

CG: control group; AT: aerobic training; ST: strength training; HE: health education.

Univariate analysis of variance.

a Significant difference versus CG, $P<.05$.

${ }^{\mathrm{b}}$ Significant difference versus $\mathrm{HE}, P<.05$.

* Significant difference versus baseline, $P<.05$.

\# Significant difference versus after intervention, $P<.05$.

B: baseline; AI: after intervention; AD: after detraining.

Mixed-model ANOVA with repeated measures.

$\$$ Significant, $P<.05$.

and functional independence is also necessary to preserve and increase muscle strength through ST [39, 40]. ST compensates the loss of muscle and bone mass associated with aging, reducing the risk of fractures, and otherwise maintains the functionality and flexibility postural stability, thus reducing the risk of falling $[41,42]$.

According to our results, we observed that ST was the most effective way to improve plasma lipoprotein lipid profiles and also improve insulin action, which results in one significant decrease of glucose, triglycerides, HDLcholesterol and TC. We observed, as Durstine et al. [43] suggested, that exercise training lasting longer than 12 weeks is more likely to increase plasma HDL cholesterol and triglycerides in a dose-dependent manner.

In this way, PA is a most appropriate intervention in a collaborative approach to the management of abnormal blood lipids and should be undertaken at moderate to high intensity, 5 to 7 days/week for at least $30 \mathrm{~min} /$ day
$[44,45]$. Furthermore, to achieve changes in lipids or weight, the majority of the studies indicate that changing dietary behaviour as well as performing vigorous exercises is fundamental and yields the best effects $[10,46]$.

Although the mechanisms underlying the lowering effect of exercise on BP are not fully understood, the recent ACSM and AHA [47] guidelines recommend light- to moderateintensity lifestyle physical activities to optimize health, and moderate or high-intensity exercise may be required to produce adaptations in the cardiovascular system and in CVD risk factors. These changes are of importance since hypertension, a common disease of older individuals, is associated with an increased incidence of all-cause and CVD mortality and morbidity such as, stroke, coronary heart disease, and renal failure [48].

Barger and Muldoon [49] concluded that hypertensive labeling was associated with poorer HRQoL as measured by global self-rated health. Kuo et al. [50] in a longitudinal 


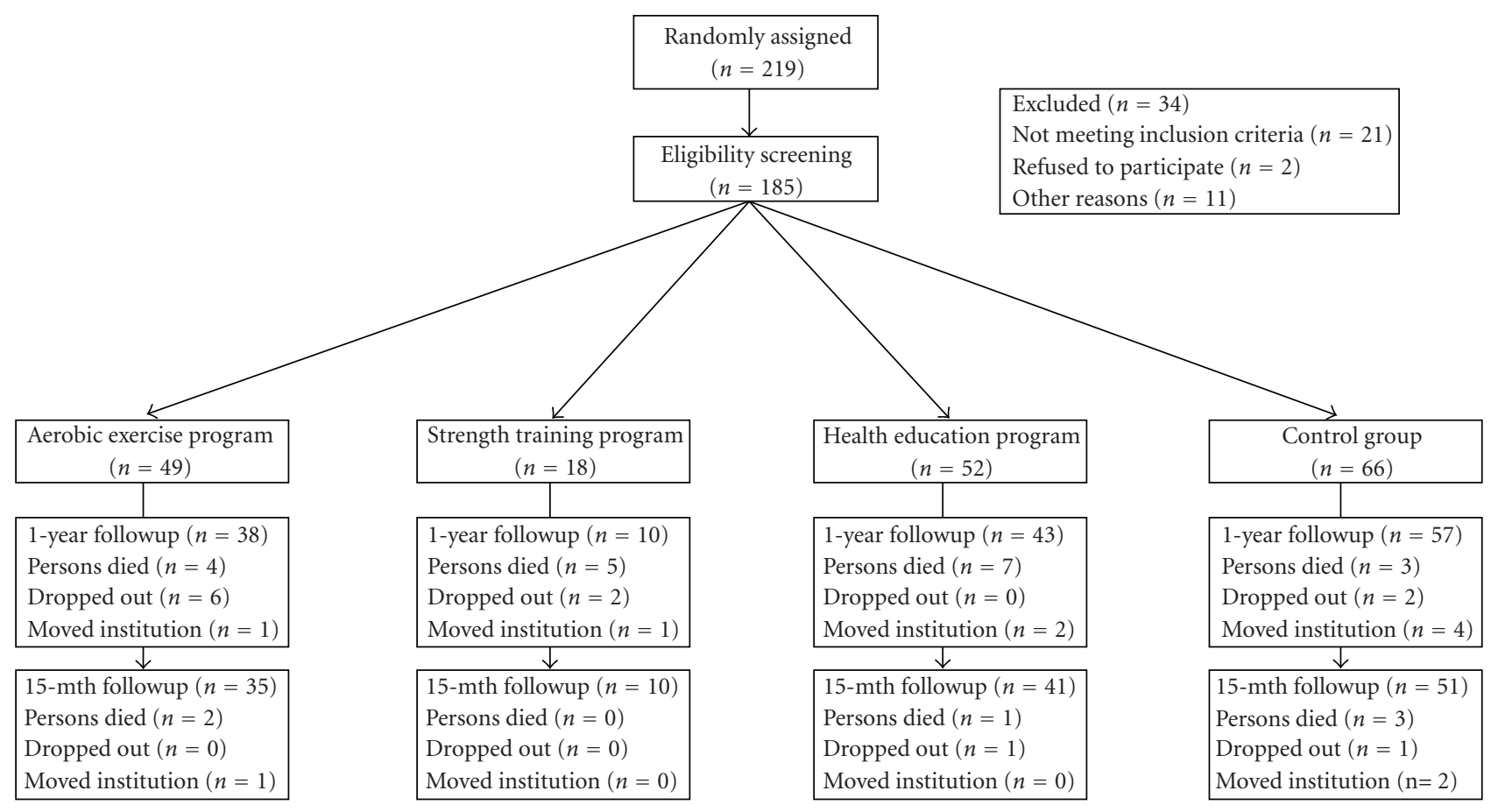

FIgURE 1: Flow diagram of enrollment, measurements.

study concluded that hypertension and diabetes were associated with a significantly faster pace of decline on the perceived HRQoL and elders with diabetes had a faster pace of decline in activities daily living functioning than nondiabetic subjects.

Many studies have examined the effects of exercise on several cardiovascular risk factors, depending on the type, intensity, duration of exercise, and participants' age and functional status and different methodologies used to evaluate older population, and results have been discordant. In fact these positive cardiovascular health changes are usually more associated with reductions of body weight and dietary fat reductions $[46,51]$. Although our subjects were instructed to maintain their normal dietary routines throughout the protocol period, this was not strictly controlled. It is possible that the participants in our study increased their energy intake in response to the energy demands of exercise training.

The data available in the literature generally support the conclusion that older adults improve their plasma lipoprotein lipid profiles with PA, increase in plasma HDL cholesterol levels, and have diminutions in triglyceride and cholesterol levels $[52,53]$. Nevertheless, these changes may be secondary to training-induced reductions in body fat scores $[54,55]$.

Nevertheless, we should highlight that our participants experienced significant changes in lipid levels. Pescatello et al. [56] showed that the existence of greater amounts of $\mathrm{PA}$ and of low intensity is associated with a more favorable lipid profile in the elderly. We also observed that higher BMI is also associated with reduced PA and functional capacity, suggesting that the risk of obesity may be explained partially by the adverse effects of inactivity. While weight loss has been shown to decrease multiple cardiovascular risk factors [57], the direct benefit of weight reduction on CVD risk remains unproven and unclear. Our data adds evidence to the role of $\mathrm{PA}$ as prevention to the CVD risk factor in elders, which is in accordance with the expected effects of health promotion intervention with PA [58].

Overall, the studies related to the risk of CVD and PA indicate that the relationship is causal and most likely to provide evidence of a dose-response relationship, showing that there is a decrease in CVD mortality in subjects with a more active lifestyle [59]. These results have been demonstrated in a variety of populations and using a diversity of methods of assessment of PA. This variability of measuring makes it difficult to compare results between studies. This sustains the need for more studies with different protocols and considering these different variables to clarify these relationships.

Our results clearly clarified the potential of an appropriate regimen of regular PA among elders, particularly with the analysis of the changes in the detraining period. Regarding the effects of detraining, like in previous studies, our results demonstrated that strength $[11,60]$ and flexibility $[13,22$, 61] gains after training decreased after 3 months of detraining, nevertheless the values remained significantly higher than before training. However, agility/dynamic balance in the AT group reverted to lower values than the baseline. In opposition, we observed that aerobic endurance was the less affected component by detraining period $[13,22]$.

Training cessation among elders is generally associated with the loss of functional ability. Some studies indicate that the morphological and functional adaptations to training may disappear, even after short periods of detraining 
$[11,62]$. Therefore, like our data support, long-term exercise programs undertaken prior to activity cessation may offer functional protection $[14,63]$. Moreover, moderate to higher intensity training may maintain the gains for longer periods after training ends.

On the other side, it is difficult to explain the discrepancy between the upper and lower body response to short-term detraining in this small sample. One possible explanation is that the initial training response was greater for lowerextremity strength than for upper-extremity strength and our subjects may have been less physically active than in some studies. Moreover, when we analyzed the results of 1year followup, it was found that elders in the control group decreased their levels of all parameters of the FFT, except for upper strength and flexibility, possibly because the muscles of the upper extremity are the most used and this could result in better physical function [42, 64]. This difference may also be explained by the activities performed in the leisure time, morphological differences in the connective and skeletal tissues, and hormonal differences [65].

According to Misic et al. [66] muscle function is the most important predictor physical function of the lower extremities in the elderly. Furthermore, aerobic endurance and fat mass are secondary predictors, which are widely associated with the functional capacity to perform various activities of daily living [6].

These underlying results show that the negative effects of detraining reduce the functional capacity of the elderly and hence reflect on their HRQoL, highlighting that PA should be practiced regularly. Supporting these results, the literature shows that the elderly who became active had a better HRQoL than their peers who remained sedentary $\mathrm{Wu}$ et al. [67], which indicates the great value of exercise interventions in the elderly.

Strengths of this study include the objective measure of PA, through 15 months of study with a 1-year intervention, controlled experimental design, and most importantly the opportunity to compare different intervention strategies. However, this study has some important limitations: first, there was no assessment of subjects' dietary regimens throughout the investigation; second, PA was not assessed during detraining and PA patterns could change during that time, due to seasonality [68]; third, there was an imbalance in group sizes. Finally, probably the main limitation of this study is that participants were selected from long-term care institutions and they may not represent all older adults, in particular those who are physically independent and community-dwelling elders. Further research is needed to determine whether diet and cardiovascular risk modification ameliorate cognitive and functional decline in elderly people.

In conclusion, results highlight that a period of 3 months of detraining following 1-year of health intervention programs significantly impairs the major part of the favorable functional changes obtained after training. Agility/dynamic balance, lower body strength, and flexibility were the components of functional fitness most affected by detraining in the AT. Therefore, our data reinforce the idea that detraining affects physical fitness and elders should be engaged in a systematic exercise program throughout life in order to maintain or improve functional performance $[69,70]$.

Thus, PA and(or) exercise prescriptions should emphasize components related to the maintenance of functional capacity and independence; these will also improve cardiovascular health.

Since low PA levels are health risk factors for ageing subjects [71], health professionals need to be more effective promoting $\mathrm{PA}$ as an integral component of a healthy lifestyle. Elders who undertake PA have better chances of maintaining or increasing physical function. Besides they can also improve their social life opportunities.

\section{Acknowledgment}

This study was supported by a grant from the Portuguese Foundation for Science and Technology (FCOMP-01-0124FEDER-009587-PTDC/DES/102094/2008).

\section{References}

[1] WHO, "World Health Organization. Physical Activity," 2007, http://www.euro.who.int/hepa.

[2] S. Karinkanta, A. Heinonen, H. Sievänen, K. Uusi-Rasi, and P. Kannus, "Factors predicting dynamic balance and quality of life in home-dwelling elderly women," Gerontology, vol. 51, no. 2, pp. 116-121, 2005.

[3] A. Lobo, J. Carvalho, and P. Santos, "Effects of healthpromotion programmes on physical activity, physical fitness and health-related quality of life of institutionalized elderly subjects: one-year results," Asian Journal of Gerontology and Geriatrics, vol. 4, pp. 8-13, 2009.

[4] P. Capodaglio, E. M. Capodaglio, A. Ferri, G. Scaglioni, A. Marchi, and F. Saibene, "Muscle function and functional ability improves more in community-dwelling older women with a mixed-strength training programme," Age and Ageing, vol. 34, no. 2, pp. 141-147, 2005.

[5] T. R. Henwood and D. R. Taaffe, "Short-term resistance training and the older adult: the effect of varied programmes for the enhancement of muscle strength and functional performance," Clinical Physiology and Functional Imaging, vol. 26, no. 5, pp. 305-313, 2006.

[6] American College of Sports Medicine, "ACSM position stand: exercise and physical activity for older adults," Medicine and Science in Sports and Exercise, vol. 30, no. 6, pp. 992-1008, 2009.

[7] J. J. Peterson, J. B. Lowe, N. A. Peterson, and K. F. Janz, "The relationship between active living and health-related quality of life: income as a moderator," Health Education Research, vol. 21, no. 1, pp. 146-156, 2006.

[8] R. Paffenbarger, "Physical exercise to reduce cardiovascular disease risk," Proceedings of the Nutrition Society, vol. 59, no. 3, pp. 421-422, 2000.

[9] T. R. Wessel, C. B. Arant, M. B. Olson et al., "Relationship of physical fitness vs body mass index with coronary artery disease and cardiovascular events in women," Journal of the American Medical Association, vol. 292, no. 10, pp. 1179-1187, 2004.

[10] R. W. Braith and K. J. Stewart, "Resistance exercise training: its role in the prevention of cardiovascular disease," Circulation, vol. 113, no. 22, pp. 2642-2650, 2006. 
[11] N. F. Toraman, "Short term and long term detraining: is there any difference between young-old and old people?" British Journal of Sports Medicine, vol. 39, no. 8, pp. 561-564, 2005.

[12] L. F. Teixeira-Salmela, L. Santiago, R. C. Magalhães Lima, D. M. Lana, F. F. Oliveira Camargos, and J. G. Cassiano, "Functional performance and quality of life related to training and detraining of community-dwelling elderly," Disability and Rehabilitation, vol. 27, no. 17, pp. 1007-1012, 2005.

[13] I. G. Fatouros, A. Kambas, I. Katrabasas et al., "Resistance training and detraining effects on flexibility performance in the elderly are intensity-dependent," Journal of Strength and Conditioning Research, vol. 20, no. 3, pp. 634-642, 2006.

[14] M. M. Porter, M. E. Nelson, M. A. Fiatarone Singh et al., "Effects of long-term resistance training and detraining on strength and physical activity in older women," Journal of Aging and Physical Activity, vol. 10, no. 3, pp. 260-270, 2002.

[15] K. Smith, K. Winegard, A. L. Hicks, and N. McCartney, "Two years of resistance training in older men and women: the effects of three years of detraining on the retention of dynamic strength," Canadian Journal of Applied Physiology, vol. 28, no. 3, pp. 462-474, 2003.

[16] N. F. Toraman and N. Ayceman, "Effects of six weeks of detraining on retention of functional fitness of old people after nine weeks of multicomponent training," British Journal of Sports Medicine, vol. 39, no. 8, pp. 565-568, 2005.

[17] J. Schlicht, D. N. Camaione, and S. V. Owen, "Effect of intense strength training on standing balance, walking speed, and sit-to-stand performance in older adults," The Journals of Gerontology: Series A, vol. 56, no. 5, pp. M281-M286, 2001.

[18] K. Häkkinen, M. Alen, M. Kallinen, R. U. Newton, and W. J. Kraemer, "Neuromuscular adaptation during prolonged strength training, detraining and re-strength-training in middle-aged and elderly people," European Journal of Applied Physiology, vol. 83, no. 1, pp. 51-62, 2000.

[19] S. P. Tokmakidis, K. A. Volaklis, and K. A. Volaklis, "Training and detraining effects of a combined-strength and aerobic exercise program on blood lipids in patients with coronary artery disease," Journal of Cardiopulmonary Rehabilitation, vol. 23, no. 3, pp. 193-200, 2003.

[20] M. F. Folstein, S. E. Folstein, and P. R. McHugh, “"Mini mental state": a practical method for grading the cognitive state of patients for the clinician," Journal of Psychiatric Research, vol. 12, no. 3, pp. 189-198, 1975.

[21] F. I. Mahoney and D. W. Barthel, "Functional evaluation: the Barthel Index," Maryland state medical journal, vol. 14, pp. 6165, 1965.

[22] M. J. Carvalho, E. Marques, and J. Mota, "Training and detraining effects on functional fitness after a multicomponent training in older women," Gerontology, vol. 55, no. 1, pp. 41-48, 2009.

[23] R. C. Wilson and P. W. Jones, "Long-term reproducibility of Borg scale estimates of breathlessness during exercise," Clinical Science, vol. 80, no. 4, pp. 309-312, 1991.

[24] R. Stella, "Bioimpedância ou Impedância Bio-elétrica," 2002, http://cyberdiet.terra.com.br/bioimpedancia-ou-impedanciabioeletrica-2-1-1-18.html.

[25] G. Sun, C. R. French, G. R. Martin et al., "Comparison of multifrequency bioelectrical impedance analysis with dualenergy X-ray absorptiometry for assessment of percentage body fat in a large, healthy population," American Journal of Clinical Nutrition, vol. 81, no. 1, pp. 74-78, 2005.

[26] P. Ferreira, "Criação da versão Portuguesa do MOS SF36: parte I: adaptação cultural e linguística," Acta Mídica Portuguesa, vol. 13, pp. 55-63, 2000.
[27] J. Ware and C. Sherbourne, "The MOS 36-item short form health survey (SF-36). I. Conceptual framework and item selection," Medical Care, vol. 30, pp. 473-483, 1993.

[28] R. E. Rikli and C. J. Jones, "Development and validation of a functional fitness test for community- residing older adults," Journal of Aging and Physical Activity, vol. 7, no. 2, pp. 129161, 1999.

[29] WHO, WHO MONICA Project. MONICA Manual, Geneva, Switzerland, 1990.

[30] R. R. Williams, P. N. Hopkins, L. L. Wu et al., Evaluación de los antecedentes familiares para prevenir la cardiopatias coronaria precoz, Am Heart Assoc-Primer de Cardiologia preventive, Barcelona, Spain, 1996.

[31] J. Reilly, L. Kelly, C. Montgomery et al., "Validation of Actigraph accelerometer estimates of total energy expenditure in young children," International Journal of Pediatric Obesity, vol. 1, no. 3, pp. 161-167, 2006.

[32] P. S. Freedson, E. Melanson, and J. Sirard, "Calibration of the computer science and applications, Inc. accelerometer," Medicine and Science in Sports and Exercise, vol. 30, no. 5, pp. 777-781, 1998.

[33] T. Marzilli, P. Schuler, K. Willhoit et al., "Effect of a community-based strength and flexibility program on performance-based measures of fitness in older AfricanAmerican adults," Californian Journal of Health Promotion, vol. 2, no. 3, pp. 92-98, 2004.

[34] R. Simons and R. Andel, "The effects of resistance training and walking on functional fitness in advanced old age," Journal of Aging and Health, vol. 18, no. 1, pp. 91-105, 2006.

[35] K. M. Winters-Stone and C. M. Snow, "Musculoskeletal response to exercise is greatest in women with low initial values," Medicine and Science in Sports and Exercise, vol. 35, no. 10, pp. 1691-1696, 2003.

[36] W. Spirduso, Physical Dimensions of Aging, vol. 31, Human Kinetics Publishing, Champaign, Ill, USA, 2005.

[37] R. H. Wood, R. E. Gardner, K. A. Ferachi et al., "Physical function and quality of life in older adults: sex differences," Southern Medical Journal, vol. 98, no. 5, pp. 504-512, 2005.

[38] American College of Sports Medicine-ACSM, ACSM's Guidelines for Exercise Testing and Prescription, Lippincott Williams \& Wilkins, Philadelphia, Pa, USA, 7th edition, 2006.

[39] M. E. Nelson, W. J. Rejeski, S. N. Blair et al., "Physical activity and public health in older adults: recommendation from the American College of Sports Medicine and the American Heart Association," Medicine and Science in Sports and Exercise, vol. 39, no. 8, pp. 1435-1445, 2007.

[40] R. W. Braith and K. J. Stewart, "Resistance exercise training: its role in the prevention of cardiovascular disease," Circulation, vol. 113, no. 22, pp. 2642-2650, 2006.

[41] H. Donat and A. Özcan, "Comparison of the effectiveness of two programmes on older adults at risk of falling: unsupervised home exercise and supervised group exercise," Clinical Rehabilitation, vol. 21, no. 3, pp. 273-283, 2007.

[42] K. Kubo, H. Kanehisa, K. Azuma et al., "Muscle architectural characteristics in women aged 20-79 years," Medicine and Science in Sports and Exercise, vol. 35, no. 1, pp. 39-44, 2003.

[43] J. L. Durstine, P. W. Grandjean, C. A. Cox, and P. D. Thompson, "Lipids, lipoproteins, and exercise," Journal of Cardiopulmonary Rehabilitation, vol. 22, no. 6, pp. 385-398, 2002.

[44] B. Fletcher, K. Berra, P. Ades et al., "Managing abnormal blood lipids: a collaborative approach," Circulation, vol. 112, no. 20, pp. 3184-3209, 2005. 
[45] B. K. Pedersen and B. Saltin, "Evidence for prescribing exercise as therapy in chronic disease," Scandinavian Journal of Medicine and Science in Sports, vol. 16, no. 1, pp. 3-63, 2006.

[46] V. Tikhonoff, E. Casiglia, A. Mazza et al., "Low-density lipoprotein cholesterol and mortality in older people," Journal of the American Geriatrics Society, vol. 53, no. 12, pp. 21592164, 2005.

[47] ACSM e AHA, "Physical activity and public health in older adults: recommendation from the American College of Sports Medicine and American Heart Association," Geriatric Nursing, vol. 28, no. 6, pp. 330-340, 2007.

[48] W. Rosamond, K. Flegal, G. Friday et al., "Heart disease and stroke statistics - 2007 update: a report from the American Heart Association Statistics Committee and Stroke Statistics Subcommittee," Circulation, vol. 115, no. 5, pp. e69-e171, 2007.

[49] S. D. Barger and M. F. Muldoon, "Hypertension labelling was associated with poorer self-rated health in the Third US National Health and Nutrition Examination Survey," Journal of Human Hypertension, vol. 20, no. 2, pp. 117-123, 2006.

[50] H. K. Kuo, R. N. Jones, W. P. Milberg et al., "Effect of blood pressure and diabetes mellitus on cognitive and physical functions in older adults: a longitudinal analysis of the advanced cognitive training for independent and vital elderly cohort," Journal of the American Geriatrics Society, vol. 53, no. 7, pp. 1154-1161, 2005.

[51] B. J. Nicklas, L. I. Katzel, J. Busby-Whitehead, and A. P. Goldberg, "Increases in high-density lipoprotein cholesterol with endurance exercise training are blunted in obese compared with lean men," Metabolism, vol. 46, no. 5, pp. 556-561, 1997.

[52] G. R. Hunter, C. J. Wetzstein, C. L. Mclafferty, P. A. Zuckerman, K. A. Landers, and M. M. Bamman, "High-resistance versus variable-resistance training in older adults," Medicine and Science in Sports and Exercise, vol. 33, no. 10, pp. 17591764, 2001.

[53] S. M. Roth, F. M. Ivey, G. F. Martel et al., "Muscle size responses to strength training in young and older men and women," Journal of the American Geriatrics Society, vol. 49, no. 11, pp. 1428-1433, 2001.

[54] D. J. Rose, "Promoting functional independence among "at risk" and physically frail older adults through communitybased fall-risk-reduction programs," Journal of Aging and Physical Activity, vol. 10, no. 2, pp. 207-225, 2002.

[55] J. Rico-Sanz, T. Rankinen, D. R. Joanisse et al., "Familial resemblance for muscle phenotypes in the HERITAGE family study," Medicine and Science in Sports and Exercise, vol. 35, no. 8, pp. 1360-1366, 2003.

[56] L. S. Pescatello, D. Murphy, and D. Costanzo, "Low-intensity physical activity benefits blood lipids and lipoproteins in older adults living at home," Age and Ageing, vol. 29, no. 5, pp. 433 439, 2000.

[57] P. Teixeira et al., "A new behavioral program to promote physical activity and long-term weight control: results from a randomized controlled trial," Medicine \& Science in Sports \& Exercise, vol. 39, no. 5, p. S12, 2007.

[58] E. Atlantis, C. M. Chow, A. Kirby, and M. A. Fiatarone Singh, "Worksite intervention effects on physical health: a randomized controlled trial," Health Promotion International, vol. 21, no. 3, pp. 191-200, 2006.

[59] S. C. Houde and K. D. Melillo, "Cardiovascular health and physical activity in older adults: an integrative review of research methodology and results," Journal of Advanced Nursing, vol. 38, no. 3, pp. 219-234, 2002.
[60] K. J. Elliott, C. Sale, and N. T. Cable, "Effects of resistance training and detraining on muscle strength and blood lipid profiles in postmenopausal women," British Journal of Sports Medicine, vol. 36, no. 5, pp. 340-345, 2002.

[61] E. Michelin, C. D. F. Coelho, and R. C. Burini, "Effects of one month detraining over health-related physical fitness in a lifestyle change program," Revista Brasileira de Medicina do Esporte, vol. 14, no. 3, pp. 192-196, 2008.

[62] C. Harris, M. DeBeliso, K. J. Adams, B. S. Irmischer, and T. A. S. Gibson, "Detraining in the older adult: effects of prior training intensity on strength retention," Journal of Strength and Conditioning Research, vol. 21, no. 3, pp. 813-818, 2007.

[63] T. R. Henwood and D. R. Taaffe, "Detraining and retraining in older adults following long-term muscle power or muscle strength specific training," The Journals of Gerontology: Series A, vol. 63, no. 7, pp. 751-758, 2008.

[64] K. Y. Z. Forrest, J. M. Zmuda, and J. A. Cauley, "Correlates of decline in lower extremity performance in older women: a 10-year follow-up study," The Journals of Gerontology: Series A, vol. 61, no. 11, pp. 1194-1200, 2006.

[65] G. J. Holland, K. Tanaka, R. Shigematsu, and M. Nakagaichi, "Flexibility and physical functions of older adults: a review," Journal of Aging and Physical Activity, vol. 10, no. 2, pp. 169206, 2002.

[66] M. M. Misic, K. S. Rosengren, J. A. Woods, and E. M. Evans, "Muscle quality, aerobic fitness and fat mass predict lowerextremity physical function in community-dwelling older adults," Gerontology, vol. 53, no. 5, pp. 260-266, 2007.

[67] C. Wu, C. Chen, W. Tsai et al., "A Randomized Controlled Trial of Modified Constraint-Induced Movement Therapy for Elderly Stroke Survivors: Changes in Motor Impairment, Daily Functioning, and Quality of Life," Archives of Physical Medicine and Rehabilitation, vol. 88, pp. 273-278, 2007.

[68] G. Plasqui and K. R. Westerterp, "Seasonal variation in total energy expenditure and physical activity in Dutch young adults," Obesity Research, vol. 12, no. 4, pp. 688-694, 2004.

[69] M. E. Cress, D. M. Buchner, T. Prohaska et al., "Best practices for physical activity programs and behavior counseling in older adult populations," European Review of Aging and Physical Activity, vol. 3, no. 1, pp. 34-42, 2006.

[70] A. Y. McDermott and H. Mernitz, "Exercise and older patients: prescribing guidelines," American Family Physician, vol. 74, no. 3, pp. 437-444, 2006.

[71] E. J. Groessl, R. M. Kaplan, W. J. Rejeski et al., "Health-related quality of life in older adults at risk for disability," American Journal of Preventive Medicine, vol. 33, no. 3, pp. 214-218, 2007. 


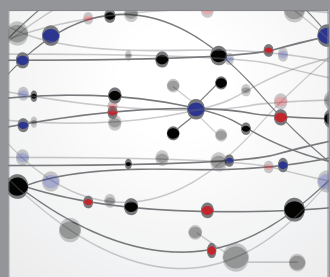

The Scientific World Journal
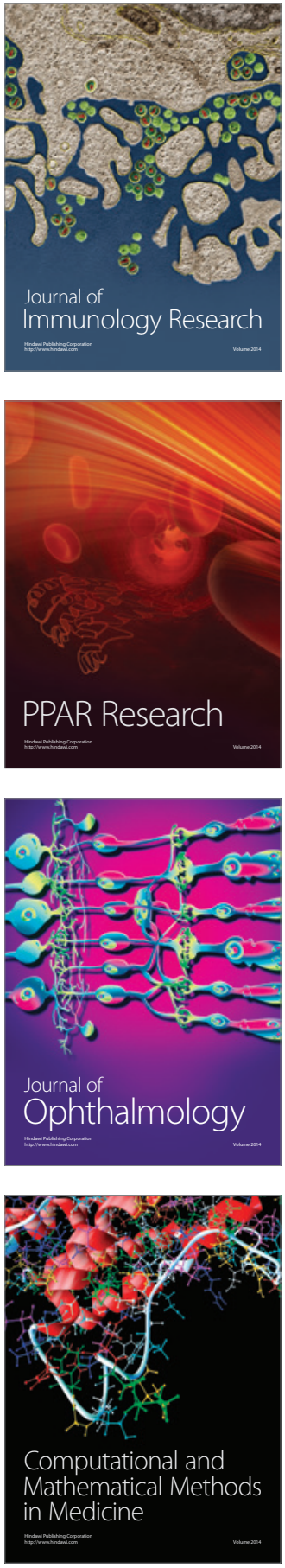

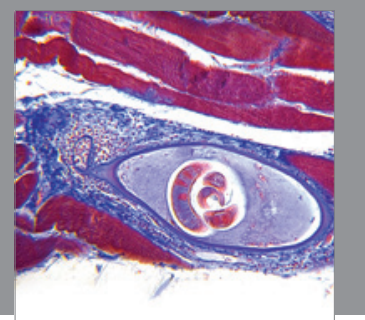

Gastroenterology

Research and Practice
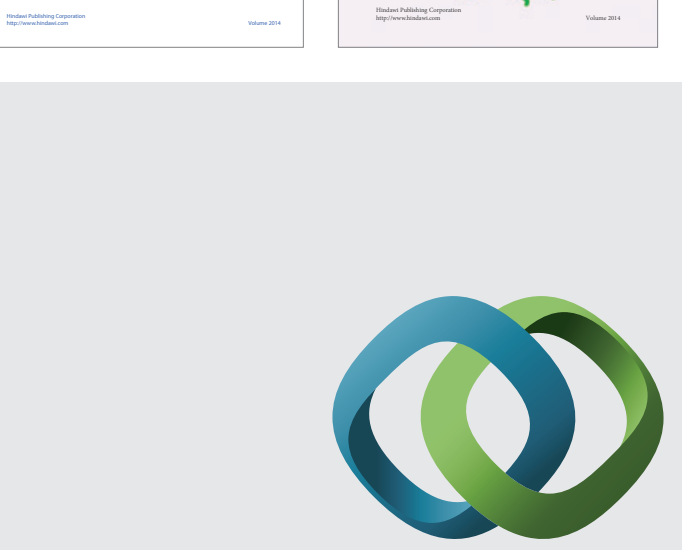

\section{Hindawi}

Submit your manuscripts at

http://www.hindawi.com
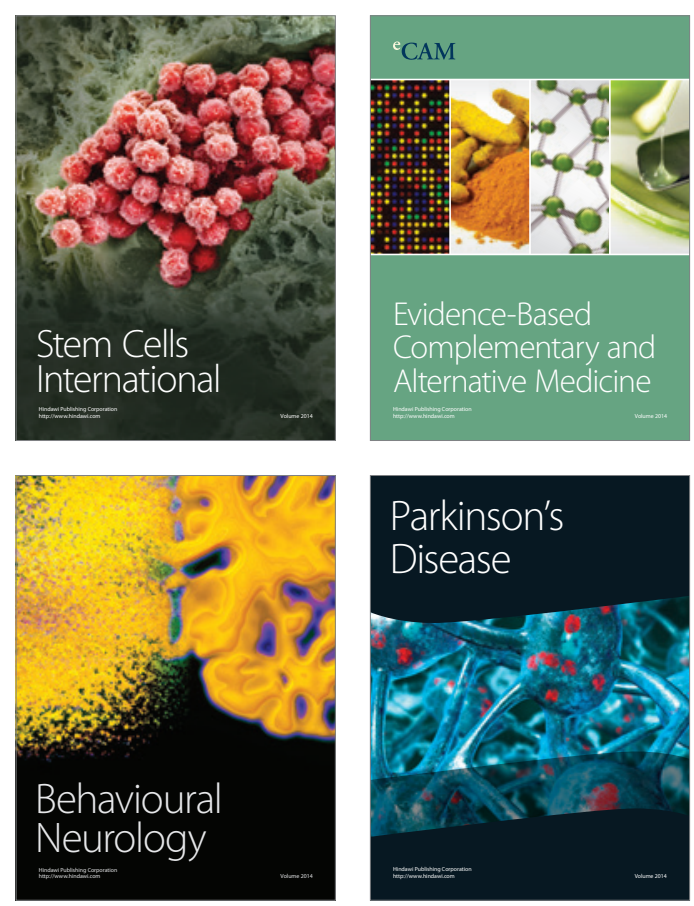

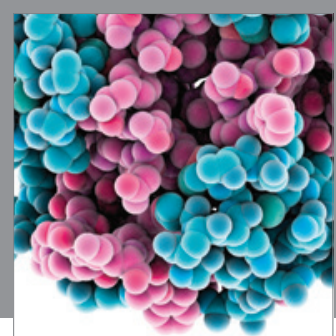

Journal of
Diabetes Research

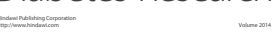

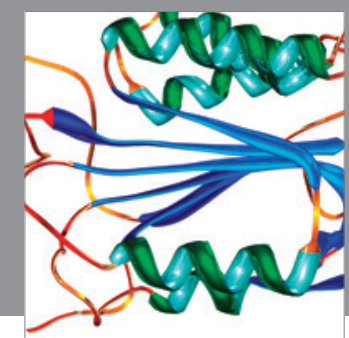

Disease Markers
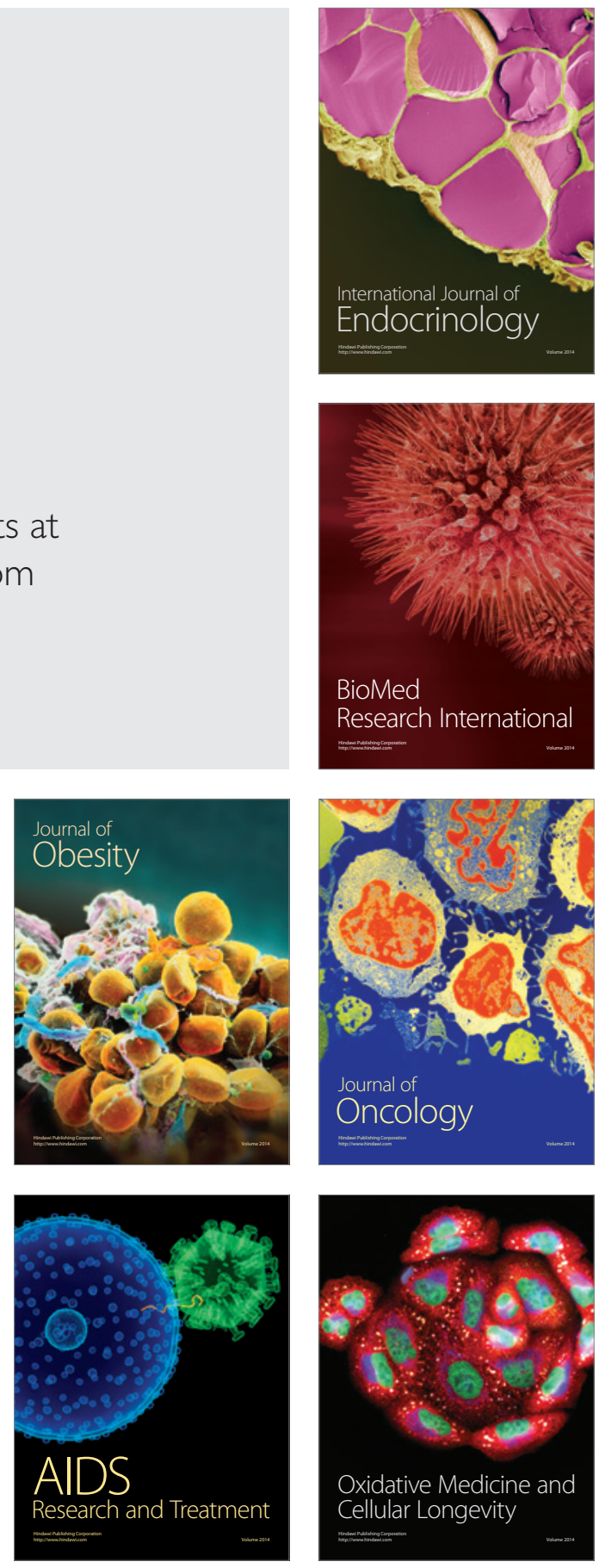